\title{
Monitoramento Remoto e Contínuo de Grandezas Elétricas Utilizando Web das Coisas e Nuvem Computacional
}

\author{
Alternative Title: Remote and Continuous Monitoring of Electrical Quantities \\ Using Web of Things and Cloud Computing \\ Marcelo Dornbusch L. \\ 4Vision Lab - MCA \\ Universidade do Vale do Itajaí \\ marcelo.cc@edu.univali.br \\ Rodrigo C. da Silva \\ 4Vision Lab \\ Universidade do Vale do Itajaí \\ rcandidosilva@gmail.com \\ Leonardo R. P. Rauta \\ 4Vision Lab - MCA \\ Universidade do Vale do Itajaí \\ leonardorauta@univali.br

Adriano M. Irigoite
Mecatrônica - DAMM
Instituto Federal de Santa
Catarina
adrianoirigoite@gmail.com \\ Paulo H. Silva \\ 4Vision Lab \\ Universidade do Vale do Itajaí \\ paulohenrique@edu.univali.br \\ Michelle S. Wangham \\ 4Vision Lab - LSED - MCA \\ Universidade do Vale do Itajaí \\ wangham@univali.br
}

\begin{abstract}
RESUMO
O monitoramento e o controle remoto de máquinas são essenciais em ambientes industriais. Tecnologias emergentes de comunicação como Web das Coisas, Comunicação Máquina a Máquina e Computação em Nuvem podem atender esta demanda de automação. O presente trabalho descreve uma solução para o monitoramento contínuo de grandezas elétricas em ambientes industriais inteligentes, que utilizam sistemas trifásicos. A solução proposta, chamada Smart Meter, utiliza uma arquitetura orientada a recursos e faz uso de um Smart Gateway para comunicação com serviços web RESTful em uma nuvem computacional. A solução proposta foi empregada em um estudo de caso real e avaliada por meio de testes de software. Os resultados obtidos nos experimentos comprovam a aplicabilidade da solução no monitoramento, bem como a corretude das medições persistidas na nuvem.
\end{abstract}

\section{Palavras-Chave}

Monitoramento Remoto de Grandezas Elétricas, M2M, Web das Coisas, Computação em Nuvem

\begin{abstract}
The remote monitoring and control of machines are essential in industrial environments. Emerging communication technologies such as Web of Things and Machine to Machine Communication can meet this demand for automation. This paper aims to introduce a solution, called Smart Meter, for continuous and remote monitoring of electrical quanti-
\end{abstract}

Permission to make digital or hard copies of all or part of this work for personal or classroom use is granted without fee provided that copies are not made or distributed for profit or commercial advantage and that copies bear this notice and the full citation on the first page. To copy otherwise, to republish, to post on servers or to redistribute to lists, requires prior specific permission and/or a fee.

SBSI 2016, May 17th-20th, 2016, Florianópolis, Santa Catarina, Brazil Copyright SBC 2016. ties, in smart industrial environments with three-phase systems. The proposed solution uses a resource-oriented architecture and makes use of a Smart Gateway for communication, RESTful web services and cloud computing. The solution was integrated with a real case study and evaluated by software testing. The results obtained demonstrate the feasibility of the solution, and the correctness of measurements persisted in the cloud.

\section{Categories and Subject Descriptors}

D.2 [Computer-Communication Networks]: Network Architecture and Design; D.1.3 [Comcurrent Programing]: [Distributed Programing]

\section{General Terms}

Network

\section{Keywords}

Remote Monitoring of Electrical Quantities, M2M, Web of Things, Cloud Computing

\section{INTRODUÇÃO}

Para a gestão eficiente de ambientes industriais, bem como o aumento do seu desempenho de produção, torna-se necessário o monitoramento de informações precisas a respeito do funcionamento das máquinas, assim como a possibilidade de atuar sobre estes equipamentos quando necessário.

Mecanismos de controle e atuação que permitam o aumento da produção são considerados de extrema importância na industria atual. Novos paradigmas, como Internet das Coisas (IoT), comunicação Máquina a Máquina (M2M) e computação em nuvem, permitem a interação de dispositivos de forma automatizada, sem ou com um mínimo de intervenção humana $[12,3]$.

$\mathrm{O}$ conceito de comunicação M2M consiste no grupo de tecnologias da informação e comunicação capazes de medir, entregar, sintetizar a informação e reagir a eventos de forma autônoma. Entre os exemplos consolidados de M2M 
estão: leituras de telemetria do sistema de freios e óleo em um carro, medições de pressão arterial e batimentos cardíacos em dispositivos médicos, monitoramento da condição de corrosão em gasodutos e oleodutos, monitoramento de consumo de água em tempo real e monitoramento e atuação em máquinas industriais [2].

$\mathrm{O}$ conceito de IoT define um paradigma que proporciona aos objetos do cotidiano a capacidade de identificação, sensoriamento, conexão e processamento, o que possibilita que estes objetos se comuniquem entre si e com serviços na Internet. Em última instância, os dispositivos da IoT têm a tendência de ser onipresentes, sensíveis ao contexto e permitem a criação de ambientes inteligentes [14].

A maioria das aplicações de IoT precisa processar e entregar uma grande quantidade de informações provenientes de um grande número de dispositivos heterogêneos. O conceito de Nuvem de Coisas (Cloud of Things - CoT), que se baseia na junção de Computação em Nuvem com a IoT, pode atender a estas demandas. A CoT usa as capacidades de virtualização da Nuvem no contexto de IoT e expõe as capacidades dos sensores e atuadores (coisas) como serviços. Na Nuvem, ocorrerão a execução de tarefas computacionais pesadas, enquanto que os dispositivos estarão focados nas tarefas de sensoriamento e atuação [11].

Estes paradigmas de comunicação, computação e atuação encontram um desafio na heterogeneidade apresentada por equipamentos no chão de fábrica, que na maioria das vezes não apresentam uma solução de interoperabilidade com outros dispositivos. Mesmo equipamentos que fornecem conexão com a Internet, muitas vezes, utilizam softwares proprietários. Para garantir a interoperabilidade nestes cenários, constata-se a tendência de tratar a IoT como Web das Coisas (WoT), no qual os padrões e protocolos da Web são empregados para prover o compartilhamento de informação $[9,15]$.

Com a finalidade de proporcionar o desenvolvimento de ambientes industriais inteligentes, projetos de pesquisa e de desenvolvimento tecnológicos estão sendo executados pela Universidade do Vale do Itajaí (UNIVALI) em parceria com a Empresa $Y^{1}$. Esta empresa possui a tradição de desenvolvimento de tecnologias inovadoras e pretende incorporar os resultados destes projetos em seus produtos e serviços.

Uma atual necessidade reportada pela empresa Y, referese ao monitoramento contínuo da qualidade da energia elétrica de suas máquinas no ambiente industrial, assim como a medição da energia consumida por estas quando em operação na cadeia produtiva de seus clientes. $\mathrm{O}$ mau funcionamento da rede elétrica do cliente pode causar uma falha na máquina industrial, o que pode gerar atraso da produção ou até mesmo causar danos ao equipamento. Para tratar este problema, a empresa $\mathrm{Y}$ dispõe em sua assistência técnica de um equipamento para realizar as medições da qualidade da rede elétrica do cliente. Diante de uma solicitação do cliente, um técnico precisa se deslocar até o cliente para realizar as medições e conduzir uma análise. Esta solução, além de não oferecer um monitoramento contínuo, o que impede a correta identificação das causas de algumas falhas, é financeiramente custosa para a Empresa Y. Além disso, a solução atual não faz a medição do consumo da máquina industrial. A medição de consumo de uma máquina industrial é muito importante para a análise econômica dos custos produtivos,

${ }^{1}$ Produtora de automação (hardware e software), para a indústria têxtil que prefere manter o anonimato. uma vez que possibilita ao cliente calcular o custo real de operação de sua máquina, por exemplo, durante a produção de uma peça.

Com intuito de atender a esta demanda, uma solução que provê o monitoramento remoto e contínuo de energia elétrica, que pode ser usada antes e após a instalação das máquinas industriais, foi desenvolvida pelas instituições parceiras. A solução proposta está baseada em um arquitetura apresentada em [6] que visa conceber um ambiente industrial inteligente. Este artigo tem como objetivo descrever esta solução de monitoramento de grandezas elétricas de máquinas industriais, chamada de Smart Meter, que se baseia no uso de Smart Gateways, Computação em Nuvem e Web das Coisas. De forma a avaliar a aplicabilidade do Smart Meter, a solução foi desenvolvida e empregada em um estudo de caso real.

O restante deste trabalho está dividido da seguinte forma. A Seção 2 analisa e compara os trabalhos relacionados. A Seção 3 apresenta a arquitetura para provimento de ambientes industrias inteligentes. A Seção 4 descreve a solução proposta para o monitoramento contínuo da energia elétrica em ambientes inteligentes. Na Seção 5, são descritos os experimentos realizados para avaliar a aplicabilidade e acurácia da solução considerando um estudo de caso. Ainda nesta seção, os resultados obtidos são analisados. Por fim, as conclusões e os trabalhos futuros são apresentados na Seção 6 .

\section{TRABALHOS RELACIONADOS}

Esta seção analisa quatro trabalhos relacionados que tratam do monitoramento remoto de ambientes industriais considerando as seguintes características: (i) se fazem uso do ambiente Web; (ii) se estes fazem uso de Serviços Web RESTful; (iii) se fazem uso de um serviço de persistência em Nuvem; (iv) se fazem uso de Smart Gateways; (v) se o Smart Gateway (SG) é embarcado; (vi) qual a tecnologia de comunicação M2M utilizada; e, (vii) se prevê o acionamento de atuadores.

Em [13], uma infraestrutura de monitoramento da qualidade da rede elétrica foi proposta. Nesta infraestrutura, são utilizados sensores anexos à rede e os dados coletados pelos sensores são enviados a um concentrador por meio de uma comunicação SPI (Serial Peripheral Interface). Este concentrador armazena os dados coletados e os disponibiliza por meio de um servidor Web embarcado; a persistência dos dados não é realizada na nuvem e a solução não faz uso de serviços Web RESTful. Os autores ainda apresentam como trabalhos futuros a implementação do protocolo IEC61850, o qual geralmente é utilizado para controle, proteção, alarme e monitoramento de subestações de energia, o que permitirá a integração com atuadores.

O trabalho descrito em [8] propõe o desenvolvimento de um sistema de monitoramento da rede de energia elétrica residencial em tempo real. Nesse trabalho, são utilizados dispositivos para o monitoramento de diferentes cargas, conectados a uma central agregadora, a qual disponibiliza as informações lidas através de um servidor Web HTTP embarcado. A comunicação entre os dispositivos e a central agregadora é feita através da utilização do protocolo CAN (Controller Area Network). O trabalho não armazena os dados coletados na nuvem e também não descreve se existe alguma persistência dos mesmos no concentrador. Além disso, o trabalho não prevê o uso de atuadores, apenas um monitoramento de baixo custo. 
Tabela 1: Comparação entre os trabalhos relacionados e a solução proposta

\begin{tabular}{c|c|c|c|c|c}
\hline & {$[13]$} & {$[8]$} & {$[5]$} & {$[4]$} & Proposto \\
\hline Ambiente Web & Sim & Sim & Sim & Sim & Sim \\
API Rest & - & - & - & Sim & Sim \\
Uso de Cloud & - & - & - & Sim & Sim \\
Smart Gateway & Sim & Sim & Sim & Sim & Sim \\
SG embarcado & Sim & Sim & Sim & - & Sim \\
Protocolo M2M & SPI & CAM & ModBus & Ethernet & ModBus \\
Prevê atuadores & Sim & - & Sim & Sim & Sim \\
\hline
\end{tabular}

Em [5], é apresentada uma arquitetura que utiliza um Gateway para disponibilizar informações obtidas através de sensores ópticos para distribuidoras de energia e gás. O trabalho foca no monitoramento de redes de distribuição de gás e os sensores ópticos são acoplados às válvulas responsáveis por essa distribuição. As informações colhidas por esses sensores são enviadas ao Gateway através de uma comunicação sem fio ponto a ponto e por meio do protocolo de comunicação Modbus, baseado em rede de sensores sem fio. O trabalho não faz uso de serviços RESTful, possui apenas um servidor web HTTP simples, também não persistem os dados na nuvem e os autores não comentam se os mesmos são armazenados no Gateway. Além disso, o trabalho não prevê a integração dessa arquitetura com alguma solução que permita o acionamento das válvulas de gás, apenas o monitoramento dessas válvulas.

Já o trabalho [4], apresenta uma arquitetura baseada em nuvem para controle e disseminação das informações coletadas de uma Smart Grid por meio de um sistema SCADA (Supervisory Control and Data Acquisition). Para efetuar o monitoramento das Smart Grids são utilizados CLPs (Controladores Lógicos Programáveis) que permitem a comunicação via Ethernet. O sistema SCADA, baseado em LabVIEW, é utilizado também como um Gateway para coletar as informações dos CLPs e enviá-las a uma plataforma na nuvem, que realiza a persistência dos dados e que os disponibiliza através de um serviço Web. Por se tratar do uso de um sistema SCADA baseado em LabVIEW, é necessária a utilização de um computador pessoal (PC) para ser utilizado como Gateway, o que faz com que a solução tenha um custo bastante elevado para ser utilizada em algumas máquinas industriais.

A Tabela 1 apresenta um comparativo entre os trabalhos correlatos e o apresentado neste artigo (Smart Meter). O trabalho de [4] é o que mais se assemelha ao aqui apresentado, uma vez que faz o monitoramento de Smart Grids e utiliza uma plataforma na nuvem para a persistência e disseminação dos dados coletados. No entanto, ele faz uso de uma solução com um custo bastante elevado. Os demais trabalhos se assemelham ao aqui apresentado por utilizarem Gateway para o monitoramento de redes elétricas e a disseminação dos dados coletados através de um ambiente web, porém não fazem uso de Nuvem e de serviços RESTful.

\section{PROVENDO AMBIENTES INTELIGEN- TES}

Muitas máquinas industriais não possuem conectividade com a Internet por meio do protocolo IP (Internet Protocol), por restrições como segurança, fatores geográficos ou custos.
Nestes casos, é possível o emprego de um dispositivo intermediário para fazer esta ligação entre máquina e a Internet, o Smart Gateway. Este dispositivo segue uma abordagem de integração de software e hardware capaz de interpretar as mensagens oriundas das máquinas e as direcionar para o destino desejado, seja uma base de dados, um servidor, ou outro dispositivo.

Em [6], os autores apresentam uma solução, baseada em uma arquitetura orientada a recursos e que faz uso de Smart Gateways e Computação em nuvem, para prover ambientes industriais inteligentes. Esta solução provê interoperabilidade entre dispositivos, persistência e processamento de dados na Nuvem e controle e monitoramento remoto via Web. A seguir, são descritos os principais componentes da solução proposta em [6] adaptados para este trabalho.

\subsection{Smart Gateway}

Um Smart Gateway é responsável por: (i) disponibilizar os recursos das máquinas monitoradas para a comunicação M2M, através de serviços Web RESTful; (ii) transmitir para um serviço Web de persistência na Nuvem os dados monitorados; e (iii) possibilitar o controle remoto de dispositivos por aplicações web hospedadas na Nuvem ou em dispositivos móveis [6]. A Figura 1 ilustra os módulos que constituem o Smart Gateway utilizado no presente trabalho.

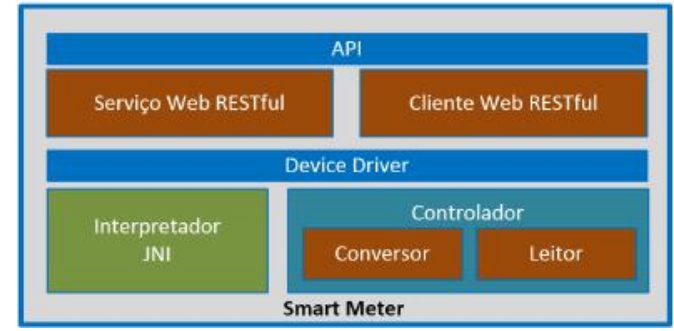

Figura 1: Visão dos módulos que constituem o Smart Gateway

O software do Smart Gateway está embarcado no hardware BBB (BeagleBone Black) ${ }^{2}$ de iniciativa open source para software e hardware. O BBB tem autonomia para funcionar de forma independente da máquina monitorada, possuindo diferentes interfaces de comunicação (Ethernet, WiFi e interfaces seriais). Estas características de comunicação possibilitam o seu uso com diferentes máquinas do setor industrial.

Incorporado ao BBB, foi utilizado uma Cape Serial, desenvolvida pela Logic Supply ${ }^{3}$, uma placa de expansão que adiciona interfaces seriais ao hardware (três portas RS232, uma porta CAN bus e uma porta RS485). Uma visão geral dos módulos de software do Smart Gateway foi apresentada na Figura 1, suas funcionalidades são descritas a seguir:

\section{* API RESTful:}

A API é composta por dois módulos: (i) Serviço Web RESTful, que disponibiliza os recursos elétricos lidos para os clientes (outras máquinas ou aplicações), o que proporciona monitoramento (via HTTP GET) e/ou controle (via HTTP POST ou PUT) destes recursos;

\footnotetext{
${ }^{2}$ http://beagleboard.org/BLACK

${ }^{3}$ http://www.logicsupply.com/cbb-serial/
} 
e (ii) Cliente Web RESTful, responsável por enviar de forma periódica os dados monitorados pelo Smart Gateway para o serviço de persistência na Nuvem;

* Device Driver:

Controladores e Interpretadores que abstraem os protocolos e APIs das máquinas no chão de fábrica, funcionando como um middleware. Este módulo se divide em: (i) Controlador, que estabelece a comunicação com o DM6200 e efetua os cálculos dos valores de potência; e (ii) Interpretador JNI, responsável por realizar o parser dos dados para a representação JSON e disponibilizar as mensagens para a APIRestful (interface de código Java/C++).

No envio dos dados feito pelo Smart Gateway para a Nuvem e para a troca de mensagens entre Smart Gateways, foi utilizado o padrão $\mathrm{JSON}^{4}$. O JSON é mais adequado para uso em sistemas embarcados por ser menos verboso e por utilizar menos recursos computacionais, quando comparado ao padrão XML ${ }^{5}[10]$.

\subsection{Ambiente de Computação em Nuvem}

O ambiente na Nuvem, implantado na plataforma Azure ${ }^{6}$ da Microsoft, é utilizada para: (i) disponibilizar um serviço Web RESTful para o recebimento de dados monitorados; (ii) persistir estes dados monitorados em bases de dados NoSQL (DocumentDB); (iii) prover uma plataforma como um serviço (PaaS), para a criação e implantação de aplicações Web de controle e monitoramento de máquinas; e (iv) provisionar aplicações de controle e monitoramento de máquinas.

Uma das aplicações que compõe o ambiente da Nuvem é denominada de Smart API, uma aplicação Web composta por serviços Web RESTful, responsáveis pelo recebimento dos dados do Smart Gateway. Para este recebimento foi desenvolvida uma abordagem assíncrona que utiliza um barramento de serviços (Enterprise Bus), gerenciado pela Azure. Este barramento é composto por uma estrutura de enfileiramento de mensagens recebidas que são encaminhadas para processos autônomos (Smart Service).

Outra aplicação que compõe o ambiente da Nuvem é denominada de Smart Service. Esta aplicação é responsável por definir os processos assíncronos da plataforma que implementa a persistência dos dados monitorados, em uma base distribuída e que provisiona o ambiente para novas aplicações. A abordagem utiliza um banco não relacional gerenciado pela Azure, denominado DocumentDB. O DocumentDB é um banco de dados orientado a documentos multi-plataforma, que utiliza um modelo de dados definido em um esquema parecido com a notação JSON, denominado BSON, o qual facilita a integração de dados em certos tipos de aplicações.

Por fim, as aplicações definidas para visualização dos dados monitorados são executadas em um ambiente isolado e virtualizado na infraestrutura da Azure. Estas foram definidas utilizando uma arquitetura Web "leve", por meio de HTML5, CSS e Javascript. Estas aplicações servem para leitura dos dados monitorados, enviando e consumindo dados de serviços REST em formato JSON definidos pela plataforma. Portanto, estas aplicações realizam consultas diretas na base de dados definida pelo DocumentDB.

${ }^{4}$ http://www.json.org/

${ }^{5}$ https://www.w3.org/XML/

${ }^{6}$ https://azure.microsoft.com/pt-br/

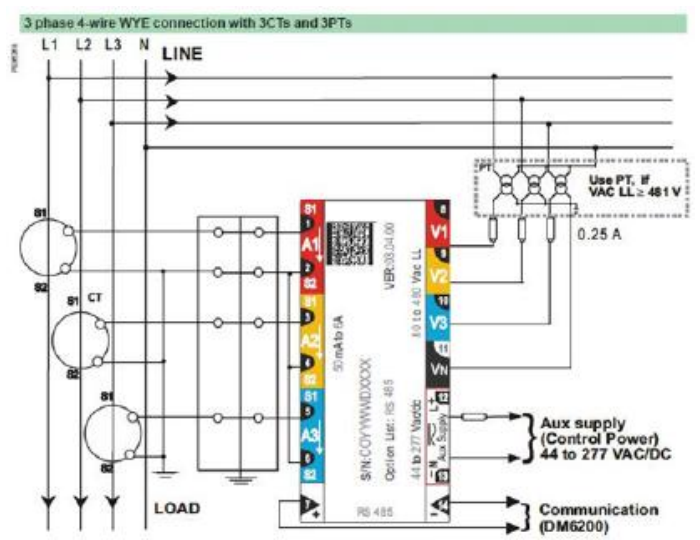

Figura 2: Esquema elétrico para aquisição dos sinais elétricos da máquina monitorada através do dispositivo DM6200

\section{MONITORAMENTO DE GRANDEZAS ELÉTRICAS}

Conforme descrito na Seção 3, a solução proposta permite o monitoramento de dispositivos heterogêneos em ambientes industriais. O presente trabalho desenvolveu uma abordagem para monitoramento contínuo de energia elétrica em equipamentos trifásicos, dando ênfase aos módulos: Controlador e Interpretador (Smart Gateway Figura 1).

O dispositivo utilizado para medir parâmetros de energia, da empresa Schneider Electric ${ }^{7}$, é um medidor digital (para sistemas trifásicos) que fornece, dentre outros, valores de tensão, corrente, fator de potência e frequência. Este medidor disponibiliza uma interface de rede RS485, com o protocolo Modbus para comunicação.

O analisador de energia é da série DM6000 da Schneider e se propõe a alta acurácia com $1 \%$ erro na leitura e flexibilidade funcional. Com funções e características para medir: (1) valores RMS de tensão fase a fase e fase e neutro; (2) correntes através dos CT's (Current Tranformer), que podem chegar a $75 \mathrm{~A}$ pico a pico na máquina monitorada da Empresa Y; (3) frequência de rede de $45 \mathrm{~Hz}$ a $65 \mathrm{~Hz}$; (4) fator de potência; (5) desbalanceamento de corrente e tensão; (6) ângulo de fase; e (7) número de interrupções no fornecimento de energia.

Estas características possibilitam uma abrangência de medidas de grandezas elétricas em sistemas trifásicos. O diagrama esquemático da Figura 2 apresenta a ligação feita entre a máquina da Empresa Y e o DM6200 para adquirir os dados da rede elétrica durante a operação da máquina.

Esta característica de comunicação do DM6200 permite integrá-lo como sensor no Smart Gateway, sendo que toda a comunicação ocorre entre o DM6200, o Smart Gateway (embarcado no hardware de baixo custo) e a arquitetura desenvolvida na Nuvem. A Figura 3 ilustra uma visão geral da abordagem proposta para monitoramento contínuo de energia elétrica, bem como a interconexão dos dispositivos e serviços.

\subsection{Módulo Controlador}

O módulo Controlador, responsável por gerenciar a troca de mensagens Modbus entre o Smart Gateway e o disposi-

$\overline{{ }^{7} \text { http://www.schneider-electric.com }}$ 


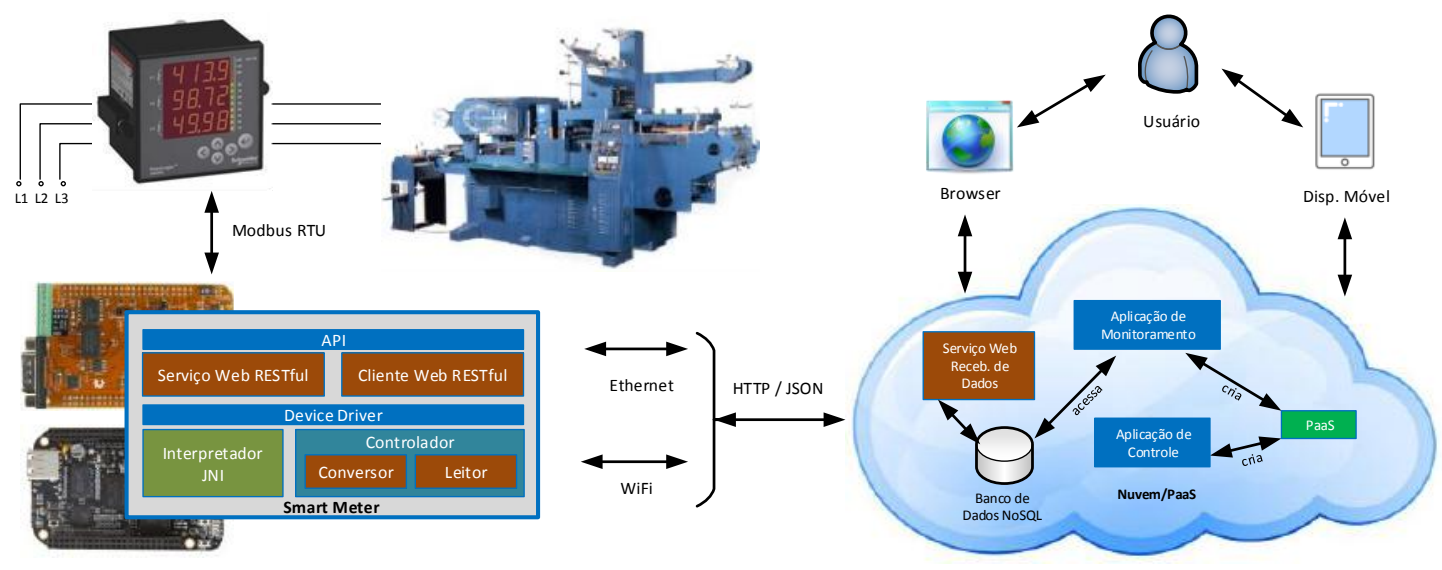

Figura 3: Visão geral do ambiente inteligente, mostra a interconexão entre os dispositivos e serviços

tivo DM6200, foi desenvolvido em $\mathrm{C}++$ e disponibilizado como uma biblioteca compartilhada (libsmartmeter.so). Quando a conexão entre ambos é concretizada, duas linhas de execução independentes são acionadas em uma abordagem produtor consumidor, disponibilizando duas funcionalidades, mostradas na Figura 1, a saber:

\section{* Leitor:}

Um processo independente, que quando inicializado dispara consultas frequentes a interface RS485 do dispositivo de leitura DM6200. Cada consulta realiza dois processos em sequência: (i) leitura, em que todo o grupo de grandezas elétricas fornecido pelo DM6200 é lido por meio do protocolo Modbus; e (ii) cálculo, no qual ocorre a computação dos valores de Potência Ativa, Potência Reativa e Aparente (relativas aos valores lidos pelo primeiro processo). Estes valores de potência não são fornecidos pelo DM6200.

Ao fim dos dois processos, um timestamp é gerado para cada consulta específica, que por sua vez é adicionada à lista de consultas disponíveis para conversão. Estas consultas são disponibilizadas para o sub-módulo Conversor.

\section{* Conversor:}

O segundo processo ${ }^{8}$, quando inicializado, consome as consultas geradas pelo sub-módulo Leitor. Cada consulta neste sub-módulo, por sua vez, sofre um processo de conversão para representação em formato JSON e é inserida em uma segunda lista. A segunda lista fica disponível para a interface JNI do módulo Interpretador.

Em suma, cada consulta disponibilizada pelo sub-módulo Controlador é composta por uma série de grandezas elétricas relativas a um timestamp. O conjunto de grandezas que compõe uma consulta é apresentado nas tabelas a seguir. Nas Tabelas 2 e 3, estão indicadas as grandezas relativas a tensão e corrente, respectivamente, e na Tabela 4, estão indicados os recursos de potência.

\footnotetext{
${ }^{8}$ Os processos Leitor e Conversor são concorrentes.
}

Tabela 2: Recursos relativos aos valores de tensão

\begin{tabular}{l|l}
\hline Recurso & \multicolumn{1}{c}{ Descrição } \\
\hline VLN & Média da tensão entre fases e neutro \\
VLL & Média da tensão entre fases \\
VUN & Desbalanceamento de tensão entre fases \\
VNN & Tensão no neutro \\
V12 & Tensão RMS entre as fases 1 e 2. \\
V23 & Tensão RMS entre as fases 2 e 3 \\
V31 & Tensão RMS entre as fases 3 e 1 \\
V01 & Tensão RMS entre a fase 1 e o neutro \\
V02 & Tensão RMS entre a fase 2 e o neutro \\
V03 & Tensão RMS entre a fase 3 e o neutro \\
VA1 & Ângulo da tensão na fase 1 \\
VA2 & Ângulo da tensão na fase 2 \\
VA3 & Ângulo da tensão na fase 3 \\
\hline
\end{tabular}

Tabela 3: Recursos relativos aos valores de corrente

\begin{tabular}{l|l}
\hline Recurso & \multicolumn{1}{c}{ Descrição } \\
\hline ALA & Média da corrente nas três fases \\
ANE & Corrente no neutro \\
AUN & Desbalanceamento de corrente nas três fases \\
A01 & Corrente RMS na fase 1 \\
A02 & Corrente RMS na fase 2 \\
A03 & Corrente RMS na fase 3 \\
PA1 & Ângulo da corrente na fase 1 \\
PA2 & Ângulo da corrente na fase 2 \\
PA3 & Ângulo da corrente na fase 3 \\
\hline
\end{tabular}

Todos os valores das três tabelas são representados por um dado do tipo real de 32 bits. Na Tabela 2, os valores são representados na unidade Volt(V). Na Tabela 3, os valores são representados na unidade Amper(A) e, por fim, na Tabela 4 os valores são representados nas seguintes unidades: Watt (W), Volt-Amper(VA), Watt Amper Reativo (VAR) e Watt/Volt-Amper(W/VA), conforme identificado na própria tabela.

As equações para o cálculo de potências ativa, reativa e aparente, utilizadas na solução proposta são apresentadas a seguir, utilizando a mesma nomenclatura apresentada nas Tabelas 2, 3 e 4. As equações relativas ao cálculo da Potência Aparente empregadas no presente trabalho são:

$$
A P 1=V 01 * A 01
$$


Tabela 4: Recursos relativos aos valores de potência (lidos e calculados)

\begin{tabular}{l|l|l}
\hline Recurso & \multicolumn{1}{c}{ Unidade } & \multicolumn{1}{c}{ Descrição } \\
\hline PFA & W/VA & Média do fator de potência nas três fases \\
ACA & W & Média da potência ativa \\
RPA & VAR & Média da potência reativa \\
APA & VA & Média da potência aparente \\
PF1 & W/VA & Fator de potência na fase 1 \\
PF2 & W/VA & Fator de potência na fase 2 \\
PF3 & W/VA & Fator de potência na fase 3 \\
TP1 & W & Potência ativa na fase 1 \\
TP2 & W & Potência ativa na fase 2 \\
TP3 & W & Potência ativa na fase 3 \\
RP1 & VAR & Potência reativa na fase1 \\
RP2 & VAR & Potência reativa na fase2 \\
RP3 & VAR & Potência reativa na fase3 \\
AP1 & VA & Potência aparente na fase1 \\
AP2 & VA & Potência aparente na fase1 \\
AP3 & VA & Potência aparente na fase3 \\
\hline
\end{tabular}

$$
A P 2=V 02 * A 02
$$$$
A P 3=V 03 * A 03
$$

$$
A P A=A C A / P F T
$$

nas quais AP1, AP2 e AP3 são o valores relativos a Potência Aparente em cada uma das três fases; V0x e A0x são o valores de tensão e corrente para cada fase, respectivamente; APA é o valor total de potência aparente; ACA é a média da potência ativa e PFT é fator de potência total.

As equações relativas ao cálculo da Potência Ativa empregadas no presente trabalho são:

$$
\begin{gathered}
T P 1=A P 1 * P F 1 \\
T P 2=A P 2 * P F 2 \\
T P 3=A P 3 * P F 3 \\
A C A=T P 1+T P 2+T P 3
\end{gathered}
$$

nas quais TP1, TP2 e TP3 são os valores de potência ativa em cada uma das três fases; PFx são os valores do fator de potência em cada uma das três fases, e ACA é o valor de potência ativa total.

As equações relativas ao cálculo da Potência Reativa empregadas no presente trabalho são:

$$
\begin{gathered}
R P 1=\sqrt{A P 1^{2}-T P 1^{2}} \\
R P 2=\sqrt{A P 2^{2}-T P 2^{2}} \\
R P 3=\sqrt{A P 3^{2}-T P 3^{2}} \\
R P A=R P 1+R P 2+R P 3
\end{gathered}
$$

nas quais RP1, RP2 e RP3 são os valores de potência reativa em cada uma das três fases e RPA é a potência reativa total. As equações empregadas para o cálculo das potências seguem a forma apresentada em [1] e [7].

\subsection{Módulo Interpretador}

O Módulo Interpretador é responsável por fazer a interface entre o código em $\mathrm{C}++$ e a API RESTful desenvolvida em Java. Ao empregar a tecnologia JNI (Java Native Interface), este módulo tem a capacidade de inicializar, controlar e receber informações do módulo Controlador.

Este módulo tem a capacidade de disponibilizar as medições através de uma interface JNI para o módulo API RESTful, responsável por enviar as consultas para serem persistidas na base não relacional da Nuvem.

\subsection{Módulo API RESTful - WSMeter}

Quando os módulos Interpretador (JNI) e Controlador estão em atividade, ocorre a geração de um buffer de leituras representadas em formato JSON (lista de consultas). Este buffer aguarda por um processo concorrente responsável pela coletada e envio a Nuvem, excecutado pela aplicação denominada WSMeter - Web Smart Meter.

WSMeter é uma aplicação web que roda no contêiner Tomcat e tem como objetivo obter medições de grandezas elétricas por meio do Módulo Interpretador e gerenciá-las até que sejam enviadas à Nuvem. Esta aplicação foi desenvolvida em Java e possui três componentes de software: (i) Schedule Manager; (ii) REST Client; e (iii) REST Service.

O Schedule Manager é responsável por fazer o agendamento das tarefas de coleta do buffer e envio para a Nuvem. Esse agendamento ocorre quando o WSMeter é inicializado pelo container web, a cada 2 s ocorre a verificação da existência de novas medidas no buffer, em caso positivo, elas são adicionadas em uma estrutura de fila. De forma concorrente, um outro processo, também agendado, verifica a cada 5 s se existem nessa estrutura de fila medições a serem enviadas para a Nuvem. Em caso positivo, o processo utiliza o módulo REST Client para o envio. As leituras gerenciadas pelo WSMeter podem ser verificadas a qualquer momento por meio de endpoints disponibilizados pelo módulo REST Service.

\section{EXPERIMENTOS E AVALIAÇÃO DOS RE- SULTADOS}

Os experimentos tiveram como objetivo avaliar a solução no que tange o (i) impacto computacional e (ii) corretude de leitura e envio dos dados obtidos para Nuvem. Desta forma o ambiente fabril teve como configuração para os experimentos, um máquina industrial munida de um medidor de energia da Schneider DM6200 conectado à solução Smart Meter e um notebook, este com o intuito de coletar informações por meio da ferramenta de Profiling JConsole.

Os recursos medidos, que formam uma string JSON, têm as seguintes dimensões: (i) 7345 caracteres; (ii) 394 palavras; e (iii) $7,46 \mathrm{~KB}$. O tempo entre leituras dos recursos monitorados, o cálculo dos valores de potência e a conversão para o formato JSON, considerando somente a aplicação em C++, é em média 472,439 ms. A medida de tempo foi efetuada no próprio código, computado tempo de CPU e tempo total para cada consulta gerada, a Tabela 5 mostra a média, a variância e o desvio padrão para um grupo de vinte 


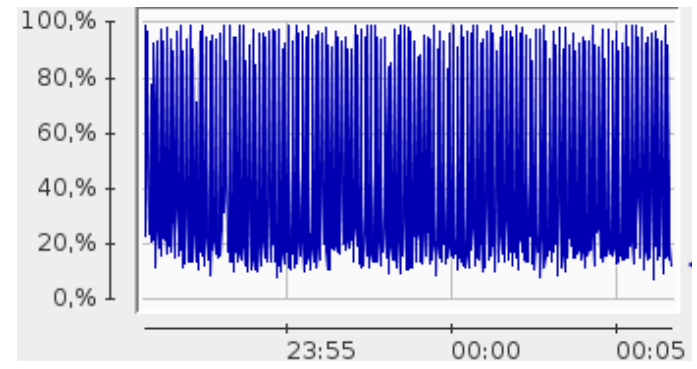

Figura 4: Consumo de CPU, porcentagem por tempo

consultas.

Tabela 5: Tempo de CPU e tempo tatal, considerando somente a aplicação $\mathrm{C}++$

\begin{tabular}{l|c|c}
\cline { 2 - 3 } & Tempo CPU(ms) & Tempo Total (ms) \\
\hline Média & 70,439 & 472,757 \\
Desvio Padrão & 0,974 & 27,190 \\
Variância & 0,950 & 739,314 \\
\hline
\end{tabular}

\subsection{Impacto Computacional}

Para a análise do impacto computacional foi utilizada a ferramenta de Profiling JConsole com o intuito de analisar o consumo de memória e CPU pela JVM durante o processo de captura, interpretação e envio das grandezas elétricas para a Nuvem. Foram analisadas as coletas em dez grupos, com intervalos 10s cada. A Tabela 6 apresenta os resultados obtidos.

Tabela 6: Média e Desvio Padrão do impacto computacional

\begin{tabular}{lc|c} 
& Uso de CPU (\%) & Uso de Memória (MB) \\
\hline Média & 24,000 & 30,400 \\
Desvio Padrão & 29,048 & 4,400 \\
\hline
\end{tabular}

O BBB tem um processador AM335x 1GHz ARM@ CortexA8, 512MB DDR3 RAM e 4GB de armazenamento flash. A pesar da variação na utilização da CPU, como pode ser observado no gráfico da Figura 4 que ilustra um intervalo de tempo monitorado, a média de consumo foi de $24 \%$. O consumo de memória por toda a solução teve uma média de 30,400 MB, o gráfico da Figura 5 ilustra um intervalo de tempo analisado, onde é possível perceber que o consumo de memória não ultrapassou 40 MB.

\subsection{Corretude das medidas}

Para a avaliação de corretude nos valores lidos, o segundo estudo de caso utilizou uma funcionalidade do DM6200, um modo de simulação que fixa valores para as gradezas elétricas disponibilizadas. A Figura 6 mostra estes valores fixos para tensão, corrente e fator de potência, respectivamente, em cada uma das três fases.

Foram conduzidos experimentos de leitura e envio para nuvem destes valores fixados no modo simulação. Três grupos de medidas foram tomadas para avaliar a corretude de

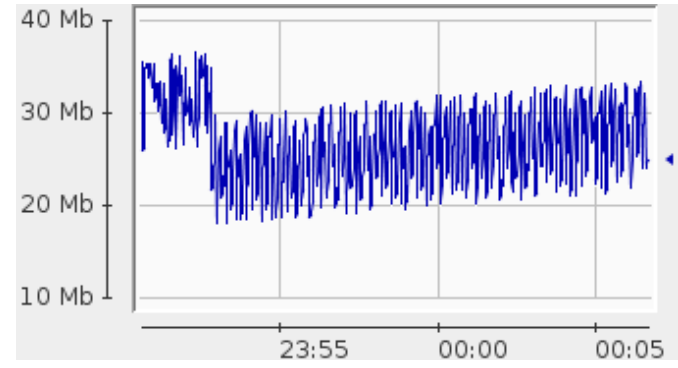

Figura 5: Consumo de Memória, MB por tempo
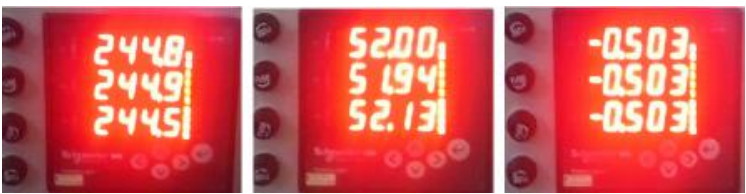

Figura 6: Valores fixados no modo simulação do DM6200, tensão, corrente e fator de potência (três fases)

leitura e persistência, em grupos de 100, 500 e 1000 mensagens. $\mathrm{O}$ valor médio, o desvio padrão e a variância para estas leituras são apresentadas nas tabelas 7,8 e 9 .

Os valores lidos foram condizentes com os observados no DM6200, sem apresentar variância ou desvio padrão nas três primeiras casas decimais. A Figura 7 ilustra o valor de tensão na fase 1 e corrente na fase 2 relativas a uma medida.

\{ "propertykey": " phase-1-voltage". "name": "Fase Voltage 1",
"description": "Fase volt "id": 55 ,

"value": "244.859".

"device": 8

\{ "propertykey": "phase-2-current" "nane": "Fase 2 corrente", "description": "Fase 2 corrente" "id": 31 , "value": "51.9414" "device":

Figura 7: Valores persistidos na nuvem, compatíveis com o observado no display do DM6200. Tensão na fase 1 e correte na fase 2 , respectivamente

Tabela 7: Valores de Tensão no experimento de leitura e envio para a Nuvem

\begin{tabular}{l|ccc}
\cline { 2 - 4 } & Tensão 1 & Tensão 2 & Tensão 3 \\
\hline Média & 244,859 & 244,915 & 244,529 \\
Desvio Padrão & 0,000 & 0,000 & 0,000 \\
Variância & 0,000 & 0,000 & 0,000 \\
\hline
\end{tabular}

Tabela 8: Valores de Corrente no experimento de leitura e envio para a Nuvem

\begin{tabular}{l|ccc}
\cline { 2 - 4 } & Corrente 1 & Corrente 2 & Corrente 3 \\
\hline Média & 52,000 & 51,941 & 52,139 \\
Desvio Padrão & 0,000 & 0,000 & 0,000 \\
Variância & 0,000 & 0,000 & 0,000 \\
\hline
\end{tabular}


Tabela 9: Valores de Fator de Potência no experimento de leitura e envio para a Nuvem

\begin{tabular}{l|ccc}
\cline { 2 - 4 } \multicolumn{1}{c}{} & FP1 & FP2 & FP3 \\
\hline Média & $-0,503$ & $-0,504$ & $-0,504$ \\
Desvio Padrão & 0,000 & 0,000 & 0,000 \\
Variância & 0,000 & 0,000 & 0,000 \\
\hline
\end{tabular}

\section{CONCLUSÃO}

A solução proposta para o monitoramento contínuo de grandezas elétricas em ambientes industriais inteligentes, que utilizam sistemas trifásicos (Smart Meter), se mostrou viável para o estudo de caso real da Empresa Y. A solução proposta segue uma arquitetura baseada em Smart Gateways para monitoramento de ambientes inteligentes e em computação em nuvem. O uso da Nuvem proporciona um infraestrutura escalável, o que possibilita alocar recursos sob demanda às aplicações Web e ao serviço de persistência de dados.

Os resultados obtidos indicam um tempo de amostragem para os valores monitorados abaixo de 500ms, o que é considerado um tempo aceitável para a leitura e cálculo das diversas grandezas elétricas e que permite o monitoramento contínuo destas grandezas. Os experimentos relativos ao impacto computacional demostraram que o hardware utilizado na proposta é capaz de suportar o processamento necessário.

As próximas etapas previstas para o trabalho dizem respeito ao aprimoramento e avaliação das aplicações web para acesso ao banco de dados não relacional, que proporcionam a visualização dos dados armazenados através de relatórios compostos por gráficos e tabelas, com opção de consultas a um intervalo de tempo específico. Com estas aplicações será possível ainda visualizar o consumo de energia elétrica em um período de tempo.

\section{AGRADECIMENTOS}

Os autores agradecem o apoio financeiro do CNPq (Projeto RHAE 459623/2013-3) e da Microsoft Research (Project: PaaS for smart machines monitoring and control).

\section{ADDITIONAL AUTHORS}

Autores Adicionais: Roni Lessa (4Vision Lab, email: ronilessa@gmail.com) and Eros Comunello (4Vision Lab MCA, email: eros.com@univali.br).

\section{REFERÊNCIAS}

[1] Ieee standard definitions for the measurement of electric power quantities under sinusoidal, nonsinusoidal, balanced, or unbalanced conditions. IEEE Std 1459-2010 (Revision of IEEE Std 1459-2000), pages 1-50, March 2010.

[2] C. Antón-Haro and M. Dohler. 1 - introduction to machine-to-machine $(\mathrm{m} 2 \mathrm{~m})$ communications. In C. A.-H. Dohler, editor, Machine-to-machine (M2M) Communications, pages 1 - 23. Woodhead Publishing, Oxford, 2015.

[3] A. Biral, M. Centenaro, A. Zanella, L. Vangelista, and M. Zorzi. The challenges of $\{\mathrm{M} 2 \mathrm{M}\}$ massive access in wireless cellular networks. Digital Communications and Networks, 1(1):1 - 19, 2015.
[4] B. Bitzer and T. Kleesuwan. Cloud-based smart grid monitoring and controlling system. In Power Engineering Conference (UPEC), 2015 50th International Universities, pages 1-5, Sept 2015.

[5] C.-H. Chen, C.-Y. Chen, C.-H. Hsia, and G.-X. Wu. Big data collection gateway for vision-based smart meter reading network. In Big Data (BigData Congress), 2014 IEEE International Congress on, pages 266-269, June 2014.

[6] M. C. Domenech, P. H. da Silva, L. R. P. Rauta, R. C. da Silva, and M. S. Wangham. Provendo um ambiente industrial inteligente com serviços web restful, smart gateways e computação em nuvem - um estudo de caso. In Anais XXXV Congresso da Sociedade Brasileira de Computação. Seminário Integrado de Software e Hardware (SEMISH), 2015, Sociedade Brasileira de Computação (SBC), 2015.

[7] A. Emanuel. Summary of ieee standard 1459: definitions for the measurement of electric power quantities under sinusoidal, nonsinusoidal, balanced, or unbalanced conditions. Industry Applications, IEEE Transactions on, 40(3):869-876, May 2004.

[8] D. Gallo, C. Landi, M. Luiso, G. Bucci, and E. Fiorucci. Low cost smart power metering. In Instrumentation and Measurement Technology Conference (I2MTC), 2013 IEEE International, pages 763-767, May 2013.

[9] S. Karnouskos, D. Guinard, D. Savio, P. Spiess, O. Baecker, V. Trifa, L. Moreira, and S. Souza. Towards the real-time enterprise: Service-based integration of heterogeneous soa-ready industrial devices with enterprise applications, 2009.

[10] N. Nurseitov, M. Paulson, R. Reynolds, and C. Izurieta. Comparison of JSON and XML Data Interchange Formats: A Case Study. In D. Che and D. Che, editors, CAINE, pages 157-162. ISCA, 2009.

[11] P. Parwekar. From internet of things towards cloud of things. In Computer and Communication Technology (ICCCT), 2011 2nd International Conference on, pages 329-333, Sept 2011.

[12] D. B. Ramamurthy. Development of a low-cost gsm sms-based humidity remote monitoring and control system for industrial applications, 2010.

[13] C. Selvam, K. Srinivas, G. Ayyappan, and M. Venkatachala Sarma. Advanced metering infrastructure for smart grid applications. In Recent Trends In Information Technology (ICRTIT), 2012 International Conference on, pages 145-150, April 2012.

[14] A. Whitmore, A. Agarwal, and L. Da Xu. The internet of things - a survey of topics and trends. Information Systems Frontiers, 17(2):261-274, 2015.

[15] D. Zeng, S. Guo, and Z. Cheng. The web of things: A survey. Journal of Communications, 6(6), 2001. 